\title{
Fuselage Boundary Layer Ingestion Propulsion Applied to a Thin Haul Commuter Aircraft for Optimal Efficiency
}

\author{
Gregor Veble Mikić*, Alex Stoll ${ }^{\dagger}$ and JoeBen Bevirt ${ }^{\ddagger}$ \\ Joby Aviation, Santa Cruz, CA, 95060, USA \\ Rok Grah \\ Institute of Science and Technology Austria, A-3400 Klosterneuburg, Austria \\ Mark D. Moore ${ }^{\S}$ \\ NASA Langley Research Center, Hampton, Virginia, 23681
}

\begin{abstract}
Theoretical and numerical aspects of aerodynamic efficiency of propulsion systems are studied. Focus is on types of propulsion that closely couples to the aerodynamics of the complete vehicle. We discuss the effects of local flow fields, which are affected both by conservative flow acceleration as well as total pressure losses, on the efficiency of boundary layer immersed propulsion devices. We introduce the concept of a boundary layer retardation turbine that helps reduce skin friction over the fuselage. We numerically investigate efficiency gains offered by boundary layer and wake interacting devices. We discuss the results in terms of a total energy consumption framework and show that efficiency gains offered depend on all the elements of the propulsion system.
\end{abstract}

\section{Nomenclature}

$\Delta \Pi_{P T}$ Change in power demand for the BLI and BLR configuration

$\Delta \Pi \quad$ Change in power demand for BLI configuration

$\delta \Pi \quad$ Change in power demand for BLR configuration

$\Delta \tilde{\Pi}_{P T}$ Change in power demand for the combination of separate BLI and BLR configurations

$\Delta p \quad$ pressure difference across the actuator,

$\eta \quad$ Propulsive efficiency

$\eta_{0} \quad$ Ideal actuator efficiency

$\eta_{a} \quad$ Apparent propulsive efficiency defined by actuator force

$\eta_{p} \quad$ Single propulsor aerodynamic efficiency

$\eta_{B L I} \quad$ BLI aerodynamic efficiency in BLI configuration

$\eta_{B L R} \quad$ BLR aerodynamic efficiency in BLR configuration

$\hat{\Pi} \quad$ BLR configuration overall power demand

$\hat{P}_{p} \quad$ main propulsion aerodynamic power in BLR configuration

$\hat{T}_{p} \quad$ main propulsion thrust in BLR configuration

$\Lambda \quad$ Lagrange multiplier for the overall horizontal force constraint

$\lambda \quad$ Lagrange multiplier of the constrained power minimization functional

$\mu_{p} \quad$ Single propulsor energy conversion efficiency

$\mu_{B L I} \quad$ BLI energy conversion efficiency in BLI configuration

\footnotetext{
${ }^{*}$ Chief Aerodynamicist, Joby Aviation, 340 Woodpecker Ridge, Santa Cruz, CA, 95060, AIAA Member.

$\dagger$ †eronautical enigneer, Joby Aviation, 340 Woodpecker Ridge, Santa Cruz, CA, 95060, AIAA Member.

${ }^{\ddagger}$ Founder, Joby Aviation, 340 Woodpecker Ridge, Santa Cruz, CA, 95060, AIAA Member.

$\S$ Aerospace Engineer, NASA Langley Aeronautics Systems Analysis Branch, Senior Member AIAA.
} 
BLR energy conversion efficiency in BLR configuration

$\mu_{p, i} \quad$ Propulsive energy conversion efficiency

$\mu_{r, i} \quad$ Regenerative energy conversion efficiency

$\nu \quad$ Velocity deficit ratio

$\omega \quad$ angular velocity of propeller or turbine

$\Pi \quad$ Overall configuration power demand

$\Pi^{\dagger} \quad$ Single propulsor overall power demand

$\sigma_{p} \quad$ Total pressure loss

$\tau \quad$ Dimensionless thrust

$\tilde{\omega} \quad$ dimensionless angular velocity of propeller or turbine

$\tilde{\omega}_{B L I}$ dimensionless angular velocity of the BLI propeller

$\tilde{\omega}_{B L R}$ dimensionless angular velocity of the BLR turbine

$\tilde{\Pi} \quad$ BLI configuration overall power demand

$\tilde{B}_{B L R}$ BLR braking force in BLR configuration

$\tilde{P}_{p} \quad$ main propulsion aerodynamic power in BLI configuration

$\tilde{T}_{p} \quad$ main propulsion thrust in BLI configuration

$\tilde{T}_{B L I} \quad$ BLI propulsion thrust in BLI configuration

$\varphi \quad$ Freestream momentum flux in front of the actuator

$\xi \quad$ Dimensionless actuator radius

$A_{0} \quad$ Far upstream area of streamtubes crossing the actuator

$B_{j} \quad$ Turbine braking component

$d \dot{m} \quad$ Infinitesimal mass flow

$D \quad$ Body drag

$d \tilde{T} \quad$ Infinitesimal thrust contribution with subtracted overall drag

$D_{E} \quad$ Equivalent drag???

$D_{e} \quad$ Second order velocity deficit entering the actuator

$D_{k} \quad$ Drag component

$D_{m} \quad$ Momentum deficit entering the actuator

$d A \quad$ Stream tube infinitesimal cross-section area at the point after which no more total pressure losses occur

$d A \quad$ Stream tube infinitesimal cross-section area

$d A_{0} \quad$ Stream tube infinitesimal cross-section area far upstream

$d A_{f} \quad$ Stream tube infinitesimal cross-section area far downstream

$d T \quad$ Infinitesimal thrust contribution

$d T^{\dagger} \quad$ Infinitesimal force on the actuator

$F \quad$ Streamwise component of total aerodynamic force

$f \quad$ Constrained power minimization functional

$F_{0} \quad$ Demanded streamwise component of total aerodynamic force

$P \quad$ Overall aircraft configuration aerodynamic power demand.

$P_{0} \quad$ Optimal power of the actuator

$p_{0} \quad$ Freestream pressure

$p_{1} \quad$ Pressure directly in front of the actuator

$p_{2} \quad$ Pressure directly after the actuator

$P_{p} \quad$ Single propulsor aerodynamic power

$P_{B L I} \quad$ BLI propulsion aerodynamic power in BLI configuration

$P_{B L R} \quad$ BLR turbine aerodynamic power in BLR configuration

$P_{p, i} \quad$ Aerodynamic power required by propulsor $i$

$P_{r, j} \quad$ Aerodynamic power generated by turbine $j$

$r \quad$ Distance from wake centre

$r_{0} \quad$ Upstream radius of the streamline furthest from the wake center

$r_{d} \quad$ Wake characteristic radius

$T \quad$ Ideal aerodynamic power

$T \quad$ Thrust

$T_{0} \quad$ Thrust constraint value

$T_{i} \quad$ Propulsor thrust component 
$v \quad$ Velocity through the actuator

$v^{\prime} \quad$ Velocity at a point after which no more total pressure losses occur

$v_{0} \quad$ Freestream velocity

$v_{0}^{\prime} \quad$ Reduced freestream velocity

$v_{f} \quad$ Downstream velocity

\section{Introduction}

Close integration of aircraft propulsion with the overall configuration of the aircraft has attracted attention in recent years. A significant number studies are concerned with efficiency improvements for airliner fuselages and the benefits that of wake ingestion, $\frac{1+6}{6}$ but application of close integration of propulsion to achieve aerodynamic benefits has also been explored for lighter aircraft ${ }^{7}$ that are operating in flight regimes closer to those of the Thin haul commuter concepts. Electric propulsion is possibly one of the enabling technologies for this research, as the scalability of electric motors in terms of power and mass allows the propulsive units to be distributed over the airframe. This allows the propulsion to become strongly integrated with the aerodynamics of the various airframe components. One particular example is wake immersed propellers $\$$ which achieve improvements in installed propulsive efficiency by imparting momentum on stagnated airflow, which reduces energy consumption, or wingtip mounted propellers whose swirl counteracts the wingtip vorticity.

Theoretical studies were performed $\sqrt{8}$ that provide the background for the efficiency gains possible with wake immersed propulsion. There remain some open questions on how such propulsion technologies are best integrated and how they benefit aircraft configurations as a whole, as is discussed in. 1 One issue that we attempt to address pertains to the interaction between the flow field around the body and the wake immersed propulsion. Flow velocity can be reduced in the vicinity of a body both due to total pressure losses as well as due to the lossless flow diffusion effects. It is not entirely known whether effects of diffusion can also be used to increase propulsive efficiency.

Another question is the one of power distribution. In a distributed propulsion system, as various propulsive units may have different optimal efficiencies and power settings at which they are observed. For a given aircraft operating point, what is the distribution of power into various propulsion devices such that the optimal efficiency is achieved? In this paper we attempt to answer the above two questions.

Furthermore, we introduce another energy device that is strongly coupled to fuselage flow, named boundary layer retardation turbine. Using CFD methods, we analyse both the turbine as well as a boundary layer ingesting propeller on an aerodynamic body corresponding to a thin haul sized aircraft. We the apply the theoretical considerations on propulsive efficiency of wake immersed propulsion to verify the consistency of results. We further analyse the obtained results within the framework of a complete aircraft configuration in order to evaluate the potential benefits of boundary layer interacting devices to overall propulsive efficiency.

\section{Boundary layer ingesting propulsor analysis}

The question of possible efficiency gains provided by propulsion ingesting the wake of a body upstream of it has been discussed in the past 810 and practical applications are utilised on ships, submarines and airships. It is, however, still somewhat unclear what benefits are available due to the body - propulsor interaction, and whether stagnation of the flow due to geometric effects and which does not reduce flow total pressure can be utilised to improve efficiency.

Here we wish to demonstrate that the efficiency of boundary layer immersed (BLI) propulsor can be boosted only on the total pressure losses in the flow upstream of it, and it does not depend on the stagnation of the flow that preserves total pressure. We then provide the ideal distribution of thrust for a BLI propulsor over the wake, which states that the wake filling should be as uniform as possible for optimal efficiency. We demonstrate the possible gains in efficiency on a wake model that allows for closed form efficiency expression as a function of propulsor thrust and diameter. For simplicity of argument incompressible flow is assumed.

\section{II.A. The case of no boundary layer interaction}

Let us first show that for a propulsor immersed in ideal flow with no total pressure losses, its theoretically maximum efficiency does not depend on the local flow picture but only on the velocity imparted onto the 
flow far downstream.

Assume the flow of fluid through an actuator plane element of infinitesimal area $d A$ aligned perpendicular to the local flow. Let the pressure jump on the element be denoted by $\Delta p$ and let the magnitude of velocity at the element be $v$. Following the stream tube up- and downstream from this element, the total change of momentum is the contribution to the overall thrust

$$
d T=d \dot{m}\left(v_{f}-v_{0}\right)
$$

where

$$
d \dot{m}=d A \rho v
$$

is the mass flow within the tube, $v_{f}$ is the velocity in the tube far downstream, and $v_{0}$ is the freestream velocity. As the flow is ideal and free of external forces except for the actuator, flow quantities within the tube are related by the Bernoulli equation that separately holds true for the flow in front, and after the actuator,

$$
\begin{aligned}
& \frac{1}{2} \rho v_{0}^{2}+p_{0}=\frac{1}{2} \rho v^{2}+p_{1}, \\
& \frac{1}{2} \rho v^{2}+p_{2}=\frac{1}{2} \rho v_{f}^{2}+p_{0},
\end{aligned}
$$

where $p_{0}$ is the freestream pressure, $p_{1}$ is the pressure in front of the actuator, $p_{2}$ the pressure just after the actuator, and $v$ is velocity at the actuator. Combining the two equations one obtains

$$
\frac{1}{2} \rho\left(v_{f}^{2}-v_{0}^{2}\right)=\Delta p
$$

where $\Delta p=p_{2}-p_{1}$ is the pressure difference across the actuator, or

$$
v_{f}-v_{0}=\frac{2 \Delta p}{\rho\left(v_{f}+v_{0}\right)} .
$$

Inserting the above into equation (1) yields

$$
d T=d T^{\dagger} \frac{2 v}{v_{f}+v_{0}}
$$

where

$$
d T^{\dagger}=d A \Delta p
$$

is thrust as experienced at the propeller plane. There exists a difference between $d T$, which is the thrust contribution experienced by the overall body and actuator combination, and $d T^{\dagger}$, which is the thrust force directly on the actuator element, which can be attributed to the interaction between the body and propeller flows and is transmitted by the pressure field.

The minimum power put into the flow to produce the thrust $d T$ is evaluated as the local thrust $d T^{\dagger}$ multiplied by the local flow velocity through the actuator,

$$
d P=d T^{\dagger} v .
$$

Propulsive efficiency can therefore be estimated as

$$
\eta=\frac{d T v_{0}}{d P}=d T^{\dagger} \frac{2 v}{v_{f}+v_{0}} \frac{v 0}{d T^{\dagger} v}=\frac{2 v_{0}}{v_{f}+v_{0}}
$$

The ideal propulsive efficiency does not depend in any way on the velocity $v$ at the actuator disk, but only on the difference between the freestream velocity $v_{0}$ and the tube downstream velocity $v_{f}$. As the overall force on an aerodynamic body depends on momentum exchange, the downstream velocity $v_{f}$ is directly related to thrust regardless of aerodynamic details in the actuator plane.

A different type of efficiency $\eta_{w}$ is obtained by simply considering thrust on the actuator $d T^{\prime}$ and not considering the interaction between the actuator and the body, namely

$$
\eta_{a}=\frac{d T^{\dagger} v_{0}}{d P}=\frac{v_{0}}{v} .
$$

$$
4 \text { of } 20
$$


If velocity $v$ at the actuator disc is reduced due to diffusion effects (while still maintaining total pressure), then the thrust on the actuator is indeed larger than it would be if the same pressure would be acting in undisturbed flow, however the pressure effect of the actuator on the nearby body that typically results in extra drag on the body in a BLI configuration must be included in order to obtain the overall efficiency result as given in equation 10 .

\section{II.B. Inclusion of pressure losses}

The analysis in the case of pressure losses that occur in the flow in front of the actuator can be handled similarly. The assumption is that the actuator does not influence the pressure losses in the flow ahead of it, although it is still allowed to interact via the pressure field with the body. Pressure losses are assumed to occur only upstream of the actuator.

Equation (3) is now written as

$$
\frac{1}{2} \rho v_{0}^{\prime 2}+p_{0}=\frac{1}{2} \rho v^{2}+p_{1} .
$$

The value $v_{0}^{\prime}$ denotes the reduced freestream velocity, which accounts for pressure losses, via

$$
\frac{1}{2} \rho v_{0}^{2}+p_{0}=\frac{1}{2} \rho v_{0}^{\prime 2}+p_{0}+\sigma_{p}
$$

where $\sigma_{p}$ denotes the overall total pressure loss from freestream to the point where the flow reaches the actuator. Equation (4) remains

$$
\frac{1}{2} \rho v^{2}+p_{2}=\frac{1}{2} \rho v_{f}^{2}+p_{0} .
$$

Combining equations 12 and 14 one gets

$$
\frac{1}{2} \rho\left(v_{f}^{2}-v_{0}^{\prime 2}\right)=\Delta p .
$$

or

$$
v_{f}-v_{0}^{\prime}=\frac{2 \Delta p}{\rho\left(v_{f}+v_{0}^{\prime}\right)} .
$$

Rewriting equation (1) as

$$
d T=d \dot{m}\left(v_{f}-v_{0}^{\prime}+v_{0}^{\prime}-v_{0}\right)
$$

leads to

$$
d T=d \dot{m}\left(\frac{2 \Delta p}{\rho\left(v_{f}+v_{0}^{\prime}\right)}-\left(v_{0}-v_{0}^{\prime}\right)\right)
$$

Until this point a single streamtube was considered. Expression (18) contains two terms, the first depends on propulsor actuation, and the second one captures drag due to pressure losses. While the second term contribution might vary for an individual stream tube as the flux $d \dot{m}$ changes with application of $\Delta p$, when integrated over all the incoming streamtubes this term evaluates to a constant and is equal to drag of the baseline configuration.

To estimate propulsive efficiency it therefore suffices to consider only the first term, namely

$$
d \tilde{T}=d \dot{m} \frac{2 \Delta p}{\rho\left(v_{f}+v_{0}^{\prime}\right)}=d T^{\dagger} \frac{2 v}{v_{f}+v_{0}^{\prime}} .
$$

As the power expression (9) remains the same, efficiency becomes

$$
\eta=\frac{d \tilde{T} v_{0}}{d P}=\frac{2 v_{0}}{v_{f}+v_{0}^{\prime}} .
$$

Again, the expression does not depend on the local flow velocity $v$ at the actuation disk, just on the velocity flow value $v_{f}$ downstream and the reduced freestream velocity $v_{0}^{\prime}$ that corresponds to pressure losses only. 


\section{II.C. Inviscid case with no regard for actuator geometry}

While the argument in subsection II.A was given for an actuator in the vicinity of a body, we can extend it without reference to the actual geometry of the propulsor or its placement. Let $d A_{0}$ be the cross section of a stream tube far upstream, and $d A_{f}$ the cross section of the same tube far downstream. The momentum flux into the tube is $\left(\rho v_{0}\right) v_{0} d A_{0}$, and the flux exiting the tube is $\left(\rho v_{f}\right) v_{f} d A_{f}$. Similarly, for energy, the flux of kinetic energy into the stream tube is $\left(\frac{1}{2} \rho v_{0}^{2}\right) v_{0} d A_{0}$, and the flux exiting the tube is $\left(\frac{1}{2} \rho v_{f}^{2}\right) v_{f} d A_{f}$. Due to incompressibility, $v_{f} d A_{f}=v_{0} d A_{0}$. Pressures are assumed equal at both ends of the stream tube. In an ideal situation one may equate the change in momentum and energy to power and thrust contributions, respectively,

$$
\begin{gathered}
d P=\left(\frac{1}{2} \rho v_{f}^{2}-\frac{1}{2} \rho v_{0}^{2}\right) v_{0} d A_{0}, \\
d T=\rho\left(v_{f}-v_{0}\right) v_{0} d A_{0} .
\end{gathered}
$$

This gives the efficiency estimate

$$
\eta=\frac{d T v_{0}}{d P}=\frac{2 v_{0}}{v_{f}+v_{0}}
$$

as before.

\section{II.D. Total pressure loss case with no regard for actuator geometry}

One may now extend the argument from section II.B to the case where there exists total pressure loss in front of the actuator. For this purpose, let us introduce a reference point in the streamline for which all the pressure losses associated with the streamline already occurred upstream, and downstream no further pressure losses occur, and only the actuator(s) can impart further changes in energy and momentum. Let velocity at this point be $v^{\prime}$ and the cross section of the streamline at that point to be $d A^{\prime}$, where $v^{\prime} d A^{\prime}=v_{0} d A_{0}$ holds due to continuity. The total change of energy from the reference point to a point far downstream is

$$
d P=\left(\frac{1}{2} \rho v_{f}^{2}+p_{0}\right) v_{f} d A_{f}-\left(\frac{1}{2} \rho v^{2}+p^{\prime}\right) v^{\prime} d A^{\prime}
$$

As in equation 12 , let the reference velocity $v_{0}^{\prime}$ be defined as

$$
\frac{1}{2} \rho v_{0}^{\prime 2}+p_{0}=\frac{1}{2} \rho v^{\prime 2}+p^{\prime}
$$

This simplifies equation 24 to

$$
d P=\frac{1}{2} \rho\left(v_{f}^{2}-v_{0}^{\prime 2}\right) v_{0} d A_{0}
$$

For total momentum exchange, one may follow (17) to write

$$
d T=\rho\left(v_{f}-v_{0}\right) v_{0} d A_{0}=\rho\left(v_{f}-v_{0}^{\prime}\right) v_{0} d A_{0}+\rho\left(v_{0}^{\prime}-v_{0}\right) v_{0} d A_{0} .
$$

The second contribution, when integrated over all stream tubes, yields the drag due to pressure losses in absence of actuation. One may therefore focus only on the first contribution and define

$$
d \tilde{T}=\rho\left(v_{f}-v_{0}^{\prime}\right) v_{0} d A_{0}
$$

Efficiency can now be calculated as

$$
\eta=\frac{d \tilde{T} v_{0}}{d P}=\frac{2 v_{0}}{v_{f}+v_{0}^{\prime}},
$$

yielding the same result as in equation (23) without any reference to the actuation method involved. 


\section{II.E. Distribution of thrust over streamlines for optimal propulsion}

The results of previous sections described theoretically attainable propulsion efficiency of individual streamtubes. The question posed in this section is what is the distribution of far downstream velocity $v_{f}$ that leads to optimal efficiency under the constraint that overall thrust is constant.

Let us assume a certain cross section $A_{0}$ of incoming streamtubes is being actuated on. Following equations (28) and (29), we may define total thrust and power as

$$
\begin{gathered}
T=\int_{A_{0}} \rho v_{0}\left(v_{f}-v_{0}^{\prime}\right) d A_{0}, \\
P=\int_{A_{0}} \rho v_{0}\left(v_{f}^{2}-v_{0}^{\prime 2}\right) / 2 d A_{0},
\end{gathered}
$$

respectively. Minimizing $P$ under the constraint $T=T_{0}$ means that the functional $f\left(v_{f}\right)=P\left(v_{f}\right)-$ $\lambda\left(T\left(v_{f}\right)-T_{0}\right)$ must be stationary with respect to variations in $v_{f}$. The Euler-Lagrange equation for this functional is simply

$$
\rho v_{f}-\lambda \rho v_{0}=0
$$

or

$$
v_{f}=\lambda v_{0} .
$$

The Lagrange multiplier $\lambda$ is determined by the constraint $T=T_{0}$. The key point is that $\lambda$ is constant regardless of the streamtube considered. This result means that the flow far downstream should be as uniform as possible across all the actuated streamtubes for optimal efficiency.

Calculating thrust with the optimal distribution of $v_{f}$ one yields

$$
T=\int_{A_{0}} \rho v_{0}\left(\lambda v_{0}-v_{0}^{\prime}\right) d A_{0}=\int_{A_{0}} \rho v_{0}\left((\lambda-1) v_{0}+v_{0}-v_{0}^{\prime}\right) d A_{0}=T_{0}
$$

or

$$
\varphi(\lambda-1)+D_{m}=T_{0}
$$

where $\varphi=A_{0} \rho v_{0}^{2}$, and

$$
D_{m}=\int_{A_{0}} \rho v_{0}\left(v_{0}-v_{0}^{\prime}\right) d A_{0} .
$$

is the momentum deficit entering the actuator. If the area $A_{0}$ captures all the streamtubes that experience pressure losses, then $D_{m}$ equals the body drag.

Inserting (33) into 31, optimal power can be expressed as

$$
P_{0}=\int_{A_{0}} \rho v_{0}\left(\left(v_{f}^{2}-v_{0}^{2}\right)+\left(v_{0}^{2}-v_{0}^{\prime 2}\right)\right) / 2 d A_{0}=v_{0}\left[\frac{\varphi}{2}\left(\lambda^{2}-1\right)+D_{e}\right]
$$

where

$$
D_{e}=\int_{A_{0}} \rho\left(v_{0}^{2}-v_{0}^{\prime 2}\right) / 2 d A_{0}
$$

is an expression that characterises the second order velocity deficit, with the dimensions of force and is related to drag. Optimal efficiency is therefore

$$
\eta_{0}=\frac{T_{0} v_{0}}{P_{0}}=\frac{T_{0}}{\frac{\varphi}{2}\left(\lambda^{2}-1\right)+D_{e}} .
$$

Using (35) to express $\lambda$, one obtains the key result

$$
\eta_{0}=\frac{T_{0}}{\frac{\varphi}{2}\left(\frac{\left(T_{0}+\varphi-D_{m}\right)^{2}}{\varphi^{2}}-1\right)+D_{e}} .
$$

This expression relates optimal efficiency to a small number of variables that depend on the wake structure, the fraction of wake captured and the thrust required. 


\section{II.E.1. A particular wake model}

For further analysis, one must assume a certain distribution of total pressure in the wake. We choose a model that leads to closed form expressions and is a reasonable approximation of actual wake structure, namely

$$
v_{0}^{\prime}(r)=v_{0}\left[1-\nu \exp \left(-\frac{\nu r^{2}}{r_{d}^{2}}\right)\right],
$$

where $r$ is the streamline distance, taken in the cross section ahead of the body, from the wake center, and

$$
\pi r_{d}^{2}=\frac{D}{\rho v_{0}^{2}}
$$

where $D$ is overall drag on the body. This last statement can be verified by inserting equation (41) into equation (36). The parameter $\nu$ is typically between 0 and 1 and expresses the maximum velocity deficit in the wake.

For this case the equations (36) and (38) have closed form expressions

$$
D_{m}\left(r_{0}\right)=\int_{0}^{r_{0}} \rho v_{0}\left(v_{0}-v_{0}^{\prime}\right) 2 \pi r d r=D\left[1-\exp \left(-\frac{\nu r_{0}^{2}}{r_{d}^{2}}\right)\right]
$$

and

$$
\begin{aligned}
D_{e}\left(r_{0}\right) & =\int_{0}^{r_{0}} \rho\left(v_{0}^{2}-v_{0}^{\prime 2}\right) / 22 \pi r d r= \\
& =D\left[1-\exp \left(-\frac{\nu r_{0}^{2}}{r_{d}^{2}}\right)-\frac{\nu}{4}\left(1-\exp \left(-\frac{2 \nu r_{0}^{2}}{r_{d}^{2}}\right)\right)\right],
\end{aligned}
$$

where $r_{0}$ is the upstream radius of the streamline furthest from the wake center streamline. We may also write

$$
\varphi\left(r_{0}\right)=\rho \pi r_{0}^{2} v_{0}^{2}
$$

which then allows us to express optimal efficiency in terms of parameters $\xi=r_{0}^{2} / r_{d}^{2}, \tau=T_{0} / D$ and $\nu$ only. Writing $\varphi(\xi)=D \xi$ one obtains the BLI efficiency model

$$
\eta_{0}(\tau, \xi, \nu)=\frac{\tau}{\frac{\xi}{2}\left(\frac{(\tau+\xi-(1-\epsilon))^{2}}{\xi^{2}}-1\right)+\left(1-\epsilon-\frac{\nu}{4}\left(1-\epsilon^{2}\right)\right)},
$$

where

$$
\epsilon(\xi, \nu)=\exp (-\nu \xi)
$$

Another consideration is that different parts of the actuator disc can work not only as propulsion but also as a turbine in this model. Per equation (33) the velocity in the slipstream of the actuator is constant in the ideal case. In order for the actuator to be working fully in the propulsive mode, the slipstream velocity should everywhere be greater than the reduced freestream velocity $v_{0}^{\prime}$. In the critical case the tips impart no additional velocity on the wake. One may therefore require

$$
\lambda v_{0}>v_{0}^{\prime}\left(r_{0}\right) .
$$

Combining expressions (35) and 41, one obtains the criterion

$$
\frac{\xi+\tau-(1-\epsilon)}{\xi}>1-\nu \epsilon
$$

that expresses the margin where the device acts in fully propulsive mode.

There also exists a regime for relatively low thrust parameters and below this actuation margin, where a part of the actuator works as a turbine (outboard) and another part as a propulsor (inboard) without any overall power gained or spent. A positive overall thrust remains, which corresponds to infinite efficiency. This means that a fully passive wake immersed propulsion device is possible that rotates freely and reduces overall drag. 
In figures 1, 2 and 3 efficiency contour plots (thin black lines marked by efficiency values) are shown for the velocity deficit parameters $\nu \in\{1,0.5,0.25\}$, respectively, as a function of the size of the actuation area parameter $\xi$ and the thrust parameter $\tau$. The thick dashed red curve denotes the margin as expressed in inequality (49), above which the actuator is working in a fully propulsive mode. The thick full blue curve denotes the regime of positive thrust at zero input power, or infinite efficiency.

It can be seen that the most important parameter that allows the BLI propulsion to attain efficiencies significantly exceeding unity is the velocity deficit parameter $\nu$, as has already been recognized $\frac{8}{8}$ Highest efficiency gains are possible when the boundary layer velocity profile has a significant velocity deficit, as can be attained in regions with sharp pressure recovery. This means that with BLI propulsion more aggressive tapering of rear fuselage might be possible with an overall gain in efficiency.

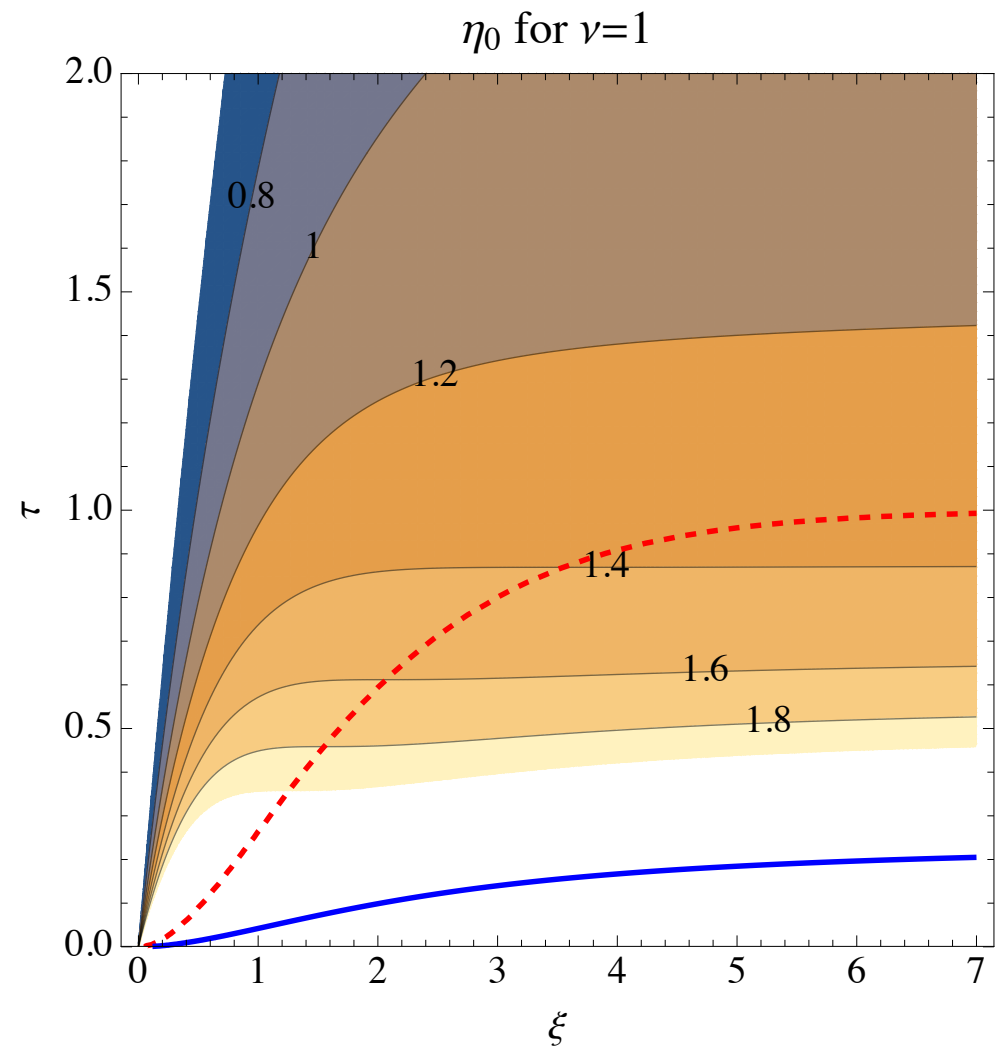

Figure 1. Efficiency contour plot for velocity deficiency parameter $\nu=1$ as a function of $\xi=r_{0}^{2} / r_{d}^{2}$ and $\tau=T_{0} / D$

\section{Overall configuration efficiency}

As seen in the discussion on wake immersed propulsion, defining aerodynamic efficiency of individual components is tricky as the interaction between the components makes it difficult to separate e.g. increase in drag versus installed propeller efficiency. If, however, one considers the overall configuration of an aerodynamic body, the overall energy efficiency is a concept that can be be uniquely defined regardless of how book keeping of various components and their interactions is performed. Furthermore, when evaluating the benefits of individual aerodynamic devices, it will be shown that these generally cannot be stated without reference to the complete configuration. This analysis follows the equivalent drag definiton as used in analysis of rotorcraft, 11 as well as the approach for evaluating overall energy efficiency in an aerodynamic configuration by focusing on power balance instead of drag and propulsion bookkeeping. 9 The analysis here is much simpler, however, as the focus is on the energy flow and associated distribution of propulsion and power generation.

One may consider an aircraft in stationary flight, such that the quantities such as drag, thrust and torque on all the components can be considered constant. For simplicity, let there be a single source of (electric) 


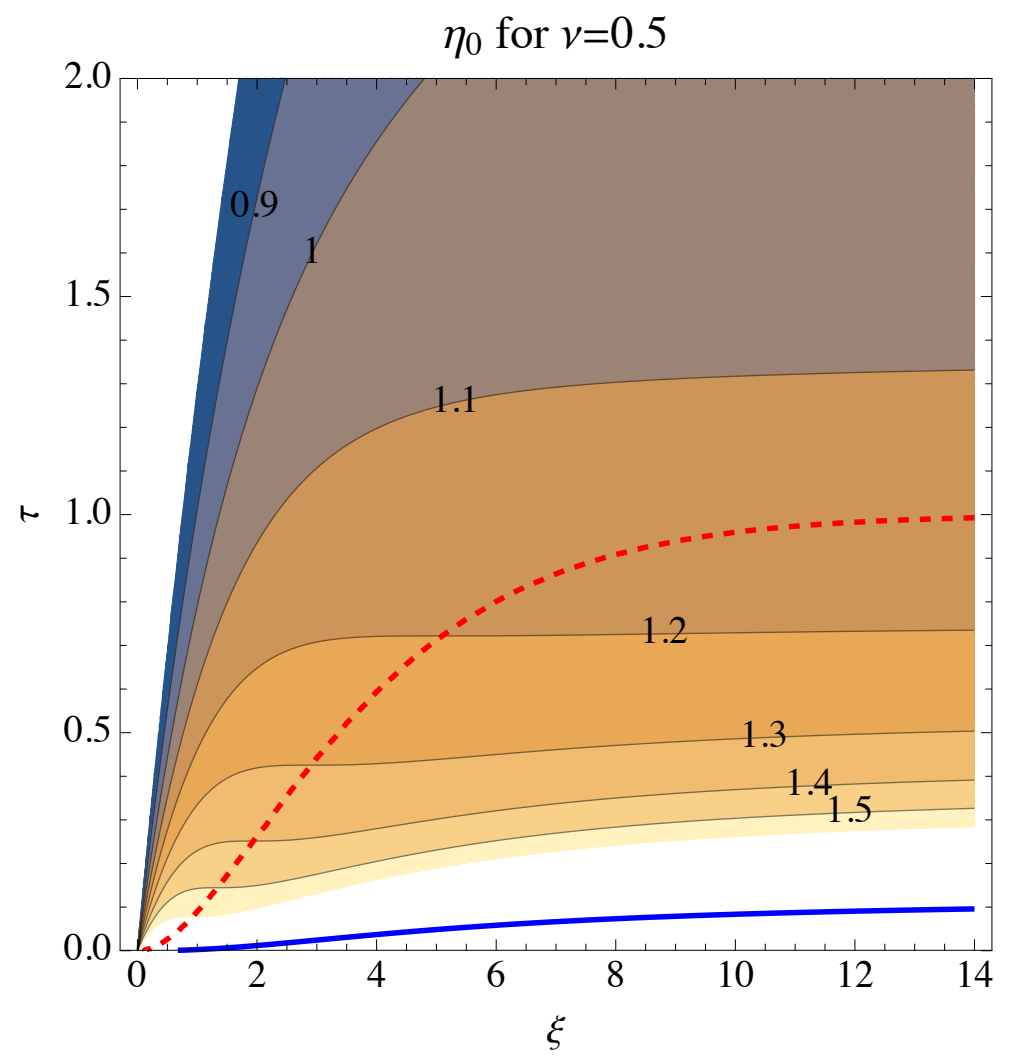

Figure 2. Efficiency contour plot for velocity deficiency parameter $\nu=0.5$ as a function of $\xi=r_{0}^{2} / r_{d}^{2}$ and $\tau=T_{0} / D$

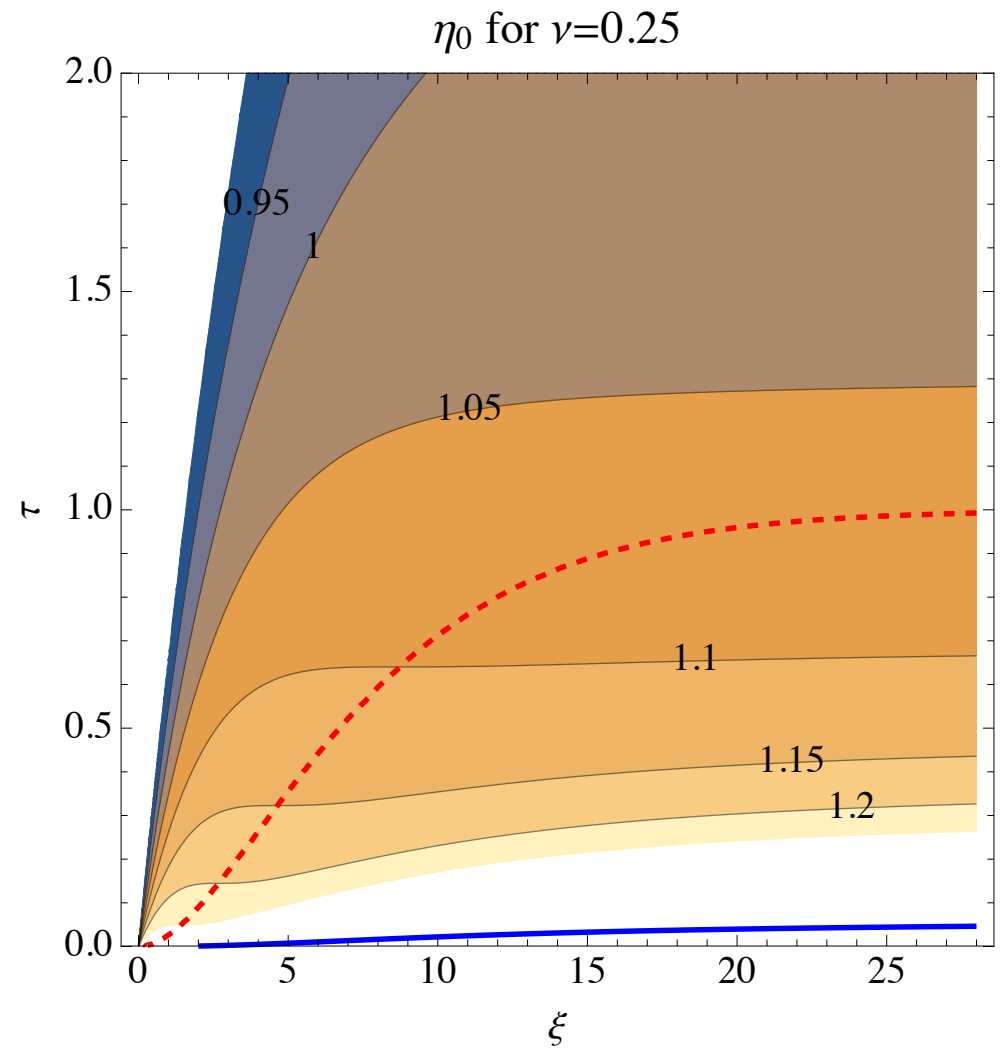

Figure 3. Efficiency contour plot for velocity deficiency parameter $\nu=0.25$ as a function of $\xi=r_{0}^{2} / r_{d}^{2}$ and $\tau=T_{0} / D$ 
energy on the aircraft that can be both spent and generated. Overall aerodynamic power demand is

$$
P=\sum_{i} P_{p, i}-\sum_{j} P_{r, j}
$$

where $P_{p, i}$ stands for the aerodynamic (shaft) power required by the propulsor $i$ and $P_{r, j}$ stands for the aerodynamic power generated by the regeneration unit (turbine) $j$, both quantities defined as positive. Denoting the efficiency of converting on board energy into aerodynamic power $\mu_{p, i}$ and the efficiency of converting aerodynamic power into on board energy as $\mu_{r, i}$, one obtains the on board power balance

$$
\Pi=\sum_{i} \Pi_{p, i}-\sum_{j} \Pi_{r, j}=\sum_{i} P_{p, i} / \mu_{p, i}-\sum_{j} P_{r, j} \mu_{r, j} .
$$

This quantity should be taken as the overall measure of energy efficiency for an electric aircraft.

Quantities such as drag and thrust do not enter the efficiency directly, but through constraints. Typically in a stationary situation, one would request

$$
F=\sum_{k} D_{k}-\sum_{i} T_{i}+\sum_{j} B_{j}=F_{0},
$$

where $F$ is the streamwise component of total aerodynamic force on the aircraft, $T_{i}$ are the thrust components from propulsors, $B_{j}$ are braking forces from turbines, and $D_{k}$ are drag components on nonmoving aircraft geometries. All individual force variables are defined as positive. $F_{0}$ is constant and is 0 in stationary forward flight, but can be different from 0 in climb or descent. Aircraft drag, propeller and turbine efficiencies therefore do not enter the power expression (51) directly, but only through the constraint (52).

The separation of total force $F$ into forces on individual components is somewhat arbitrary, as one can take the pressure interaction between e.g. a propeller and body and add it to the respective components to obtain installed propeller thrust, but the overall force on the configuration of course remains independent of the definitions. Consumed electric power, is, however, independent of the thrust and drag definitions. For horizontal flight, this allows one to define equivalent drag ${ }^{11}$

$$
D_{E}=\frac{\Pi}{v}
$$

as a measure of efficiency.

A simple instructive example is the one of a single propulsor in horizontal flight. Equation (51) is simply

$$
\Pi^{\dagger}=\frac{P_{p}}{\mu_{p}}
$$

and expression 52 becomes

$$
T_{p}=D
$$

If one defines propulsor efficiency as

$$
\eta_{p}=\frac{T_{p} v}{P_{p}}
$$

where $v$ is the forward velocity, one obtains

$$
\Pi^{\dagger}=\frac{v D}{\eta_{p} \mu_{p}} .
$$

In order to minimize power losses, the product of propeller and energy conversion efficiencies should be maximal, as expected. Note, however, that the definitions of $T_{p}$ and $D$ are not unique. On top of possible scrubbing losses, there can also exist a pressure interaction between the propulsor and the body. The value of efficiency $\eta_{p}$ changes in accordance with the definition of $T_{p}$. One option is, for example, to define drag to be independent of applied propeller power $\Pi$ and equal to the drag on the body in a configuration with no propulsion, $D_{0}$. Then thrust can be defined to be equal to $D_{0}$ in horizontal flight, and $\eta_{p}$ then represents the installed efficiency that takes into account both scrubbing drag as well as pressure interactions. 


\section{III.A. Evaluating benefits of boundary layer immersed propulsion in an overall configuration}

While the single propulsor configuration analysis yielded expected results, the situation becomes more involved when two sources of propulsion exist on the same aircraft. Equation (51) in horizontal flight becomes

$$
\tilde{\Pi}=\frac{\tilde{P}_{p}}{\mu_{p}}+\frac{P_{B L I}}{\mu_{B L I}}
$$

and expression $(52)$ is

$$
\tilde{T}_{p}+T_{B L I}=D
$$

where the index $p$ corresponds to the main propulsion unit, and $B L I$ denotes the boundary layer immersed propulsor (but could in principle be any alternative mode of propulsion). We may compare this configuration to the basic propulsor configuration in equations (54) and (55). Assuming constant propulsor efficiencies $\mu_{p}$ and $\eta_{p}$, the difference

$$
\Delta \Pi=\tilde{\Pi}-\Pi^{\dagger}=\frac{\tilde{P}_{p}-P_{p}}{\mu_{p}}+\frac{P_{B L I}}{\mu_{B L I}}
$$

becomes

$$
\Delta \Pi=v\left[\frac{\tilde{T}_{p}-T_{p}}{\mu_{p} \eta_{p}}+\frac{T_{B L I}}{\mu_{B L I} \eta_{B L I}}\right] .
$$

Due to the constraints (59) and (55), and assuming that drag $D$ is defined as constant for both configurations, this is further simplified to

$$
\Delta \Pi=v T_{B L I}\left[\frac{1}{\mu_{B L I} \eta_{B L I}}-\frac{1}{\mu_{p} \eta_{p}}\right] .
$$

The expression shows that there can be benefit (negative $\Delta \Pi$ ) from the BLI propulsor only as long as the efficiency of the BLI propulsor is higher than the efficiency of the other mode of propulsion, namely

$$
\mu_{B L I} \eta_{B L I}>\mu_{p} \eta_{p} .
$$

If one considers tip mounted propellers, for which the installed efficiency can be significantly above unity, then utilizing the BLI propulsion is ineffective, even if efficiency of the BLI propulsor is above unity, unless it is more efficient than the main mode of propulsion. This of course assumes that aerodynamic efficiency is the only criterion.

\section{III.B. Boundary layer retardation turbine in an overall configuration}

A boundary layer retardation (BLR) turbine is a device mounted on the nose of the aerodynamic body which removes the energy from the air that forms the boundary layer further downstream. This reduces skin friction on the body, and while the turbine provides a braking force, it also generates power, so there is possibility for the turbine to have positive overall energy efficiency gains.

In this example, the power spent is equal to

$$
\hat{\Pi}=\frac{\hat{P}_{p}}{\mu_{p}}-P_{B L R} \mu_{B L I} .
$$

and expression $(52)$ is

$$
\hat{T}_{p}=D+B_{B L R},
$$

where $B_{B L R}$ is the braking force on the turbine. Similarly to the case of the BLI propulsor, the change in overall configuration power can be expressed as

$$
\delta \Pi=\hat{\Pi}-\Pi^{\dagger}=\frac{\hat{P}_{p}-P_{p}}{\mu_{p}}-P_{B L R} \mu_{B L I}
$$

Defining the aerodynamic efficiency of the BLR turbine as

$$
\eta_{B L R}=\frac{P_{B L R}}{v B_{B L R}}
$$


which one expects to be above unity if the basic idea has merit, one obtains

$$
\delta \Pi=v\left[\frac{\hat{T}_{p}-T_{p}}{\mu_{p} \eta_{p}}-B_{B L R} \mu_{B L R} \eta_{B L R}\right] .
$$

After also considering expression 65, the expression is simplified to

$$
\delta \Pi=-v B_{B L R}\left[\mu_{B L R} \eta_{B L R}-\frac{1}{\mu_{p} \eta_{p}}\right] .
$$

This expression states that power balance is indeed improved the higher the combined power conversion and aerodynamic efficiency of the BLR turbine is, however the benefits may be diminished by the inefficiency of the propulsion part of the system, as expressed by the second term in the brackets. On the other hand, if propulsive efficiency is above unity, such as is the case with tip mounted propellers, then it makes the effect of BLR turbine that much more beneficial, as the braking force on the turbine can be offset by efficient generation of extra thrust needed to overcome braking. This is in contrast to BLI propulsion whose benefits are reduced with increasing propulsive efficiency of the main propulsion. Even if the efficiency of the turbine is below unity, benefits can still be had if propulsive efficiency is above unity.

\section{III.C. Optimality of distributed propulsion}

The above examples can be extended to a more general discussion. If one is to minimize power consumed in equation (51) under the constraint 52 with respect to individual consumed or generated power variables $\Pi_{p, i}$ and $\Pi_{r, j}$, respectively, one needs to find the stationarity of the expression

$$
\Pi+\Lambda F,
$$

where $\Lambda$ is the Lagrange multiplier for the overall horizontal force constraint, by requesting

$$
\frac{\partial}{\partial \Pi_{p, i}}[\Pi+\Lambda F]=0
$$

and

$$
\frac{\partial}{\partial \Pi_{r, j}}[\Pi+\Lambda F]=0 .
$$

Assuming that for each propulsion or regeneration unit the thrust and power relationships are independent of the other units, this leads to

$$
\frac{\partial F}{\partial \prod_{p, i}}=-1 / \Lambda
$$

and

$$
\frac{\partial F}{\partial \Pi_{r, j}}=1 / \Lambda
$$

Expression (73) states that, in order to optimally distribute propulsion on all the propulsion units of the aircraft, they all have to be running at the same overall differential energy efficiency $1 / \Lambda$, namely that they have to be operating at a setting where they all consume the same amount of infinitesimal on board power per infinitesimal variation of overall propulsive force. According to expression (74), for any turbines mounted on the aircraft, they should be operating at the point where their differential efficiency equals $\Lambda$, if efficiency is defined as the (differential of) amount of on board power produced per (differential of) braking force times velocity. The same value of $\Lambda$ applies in the case where both propulsors and turbines are present on the aircraft.

\section{Boundary layer retardation turbine and BLI propeller CFD analysis}

We performed numerical studies of an a BLI propeller and a BLR turbine design in order to validate the efficiency considerations of the previous section. The fuselage was sized to be representative of a thin haul commuter aircraft with up to 12 seats. For simplicity, it was chosen to be a body of revolution with 
a radius of $0.85 \mathrm{~m}$ and the length of $12 \mathrm{~m}$. The designs for the propeller (radius of $0.232 \mathrm{~m}$ ) and turbine (radius $0.27 \mathrm{~m}$ ) were iterated both in terms of blade planform, twist, radius and the number of blades such that their interaction with the fuselage boundary layer interaction was deemed favourable, but no actual optimisation was performed. The goal is to demonstrate that efficiency gains are possible with further scope for improvement.

The method used for propeller and turbine analysis was a stationary incompressiblec alculation with multiple rotating reference frames. The code used was the OpenFOAM v3.0+, and cfMesh v1.1.1 was used to generate the octree-based hex-dominant meshes with boundary layers. The application used was simpleFoam using the k- $\omega$ SST turbulence model with wall functions. The Reynolds number chosen was $4 \cdot 10^{6}$ for the reference length of $l=1 \mathrm{~m}$.

Some renderings of the CFD computations of the considered geometry with both the BLI propeller as well as the BLR turbine are shown in figure 4. In the computational case shown, both the propeller and turbine are spinning with the dimensionless angular velocities $\tilde{\omega}=\omega l / v_{0}=10$, if $v_{0}$ is aircraft forward velocity and $\omega$ is angular velocity. We also show the reduced velocity that corresponds to total pressure, namely $v_{0}^{\prime}=\sqrt{v^{2}+2\left(p-p_{0}\right) / \rho}$.

The comparison between actual flow velocity (upper half of plots) and reduced flow velocity (lower half), deliberately shown using the same scale and colours, clearly shows the distinction between flow stagnation due to fuselage flow blockage and diffusion, and the total pressure losses due to skin friction. Flow stagnation at the nose and diffusion at the tail of the fuselage clearly affect a significantly larger volume than just the total pressure losses, however those areas cannot be utilised by the BLI propeller in order to boost its efficiency.

The BLR turbine reduces the flow velocity in the boundary layer near the nose of the aircraft with no apparent significant adverse effects on the pressure recovery at the tail for this particular geometry. The BLI propeller can be seen to fill the wake velocity deficit fairly uniformly, which is what is required for efficient operation.

Numerical irregularities can be observed in the velocity flow fields near the boundaries of the rotating frame zones, which can be attributed to the non-uniformity of flow that violates the assumption of axial symmetry at the boundary. A non-stationary analysis with rotating meshes would solve this issue, but it would also significantly increase computational costs. The level of fidelity was deemed to be sufficient with a multiple reference frame approach for the purposes of demonstration of effects. Further design optimisation would likely require a higher fidelity approach.

The CFD calculations were performed for a pure fuselage, fuselage with only the BLR turbine, fuselage with only the BLI propeller, and fuselage with both the BLR turbine and the BLI propeller. In all cases, the rotating reference frame zones for both the turbine and the propeller were included in the calculation in order to make results more comparable.

As a first analysis, fuselage with the BLI propeller was considered in comparison to the baseline pure fuselage. Drag was computed for a configuration without the propeller. With propeller installed, and any changes in the overall forward force are considered attributed to propeller thrust. Aerodynamic forces and moments were evaluated for different dimensionless propeller angular velocities $\tilde{\omega}_{B L I} \in\{5,6,7, \ldots, 13\}$. The resulting propeller efficiency results, defined as the change in horizontal force from the baseline configuration times velocity, divided by shaft power, are given in figure 5. The full curve represents the numerical CFD results, and the red curve gives the estimate of the theoretical propeller efficiency as outlined in equation (46) for parameters $\xi=2.2$, which was estimated from propeller diameter and fuselage blockage effects, the velocity deficit parameter $\nu=0.5$, and an additional figure of merit factor of 0.74 with which the efficiency was multiplied to fit the data. The increasing discrepancy between the model and CFD data when approaching 0 loading on the propeller is due to skin friction losses on the blades.

As discussed in subsection (III.A), one may define energy efficiency through overall power consumption using expression (61). The power consumption benefits depend not only on the BLI propeller, but the efficiency of the main mode of propulsion. The effect of efficiency of the main mode of propulsion on power ballance expressed as a fraction of baseline power requirement $\Pi_{0}=D v$, is shown in figure 6. The energy conversion efficiency was chosen as $\mu_{B L R}=1$ in order to show pure aerodynamic benefits, but of course in a realistic installation benefits would be reduced. The less efficient the main mode of propulsion is, the more benefit one can get from the BLI propeller. There may be no benefit if the main power propulsion is more efficient than the BLI propeller.

Similarly, the BLR turbine was considered without the BLI prop initially. Again, the configuration 


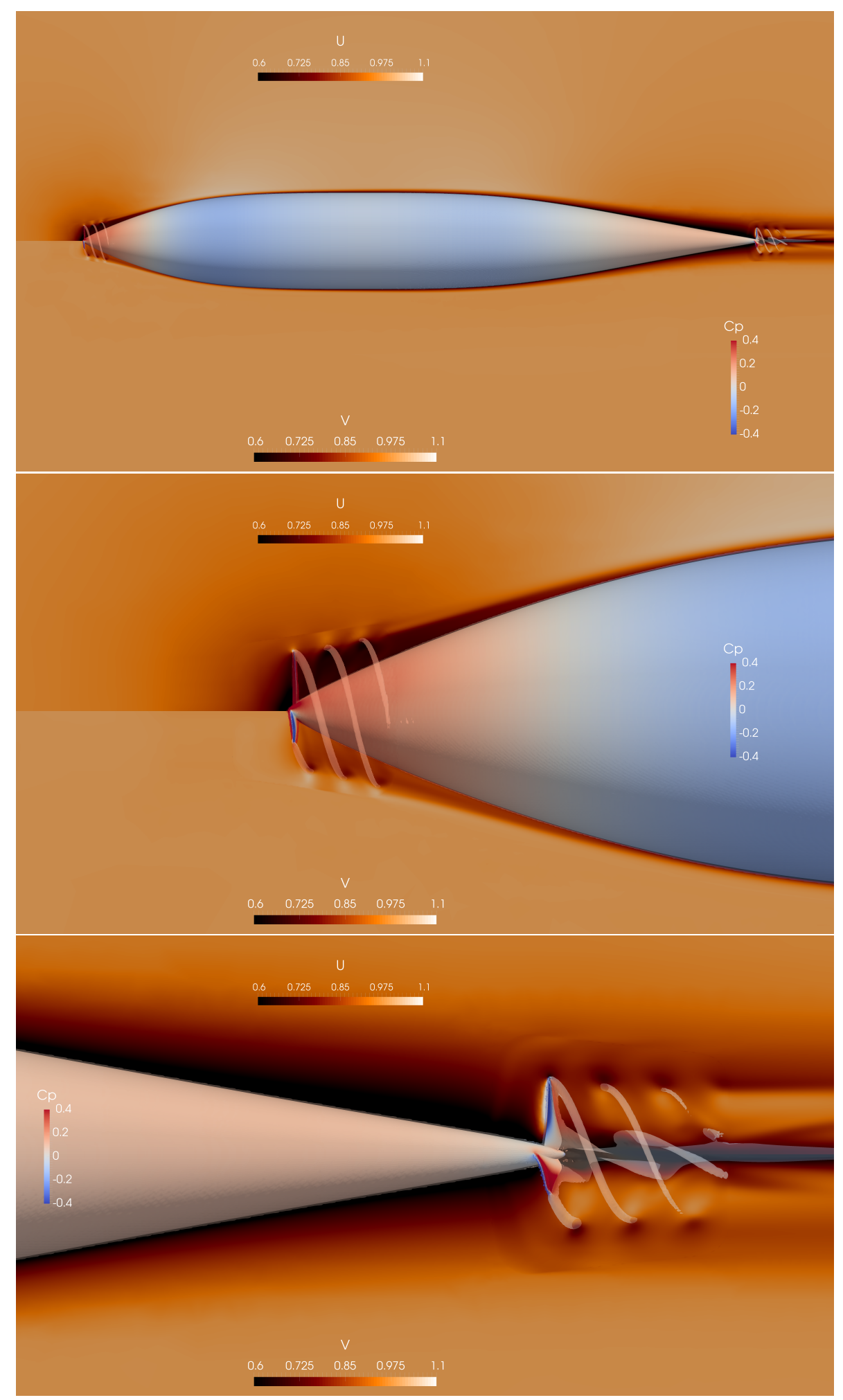

Figure 4. Normalized velocity magnitude $U=v / v_{0}$ (upper half), reduced normalized freestream velocity $V=v^{\prime} / v_{0}$ (lower half, see text) and pressure coefficient $C_{p}=\frac{p-p_{0}}{\rho v_{0}^{2} / 2}$ plot of the BLR turbine and BLI propeller setup, togxr with a contour of constant vorticity that indicates blade tip votices. 


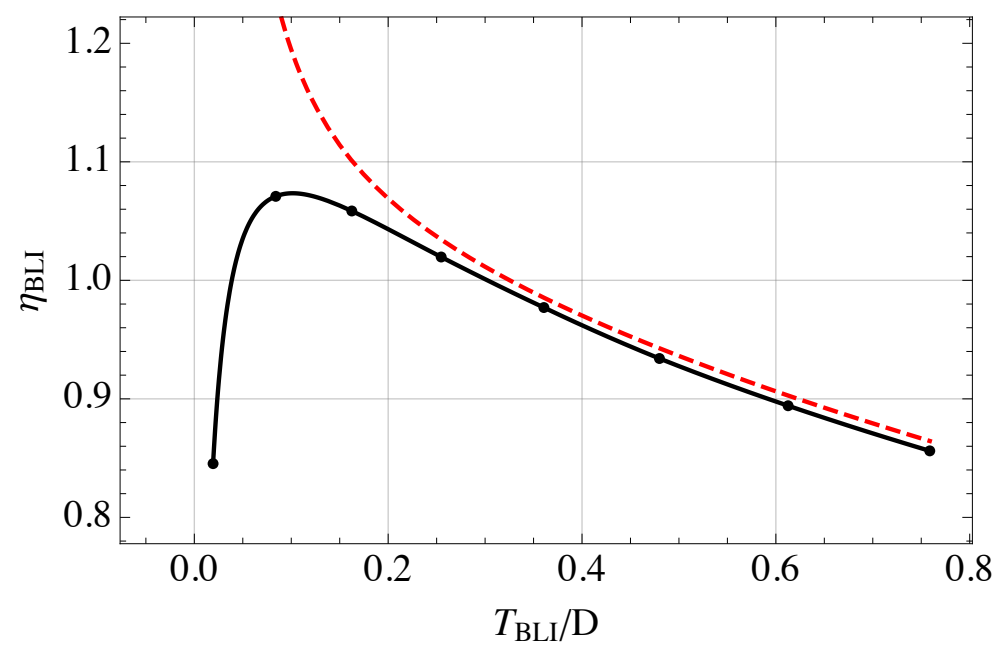

Figure 5. CFD computed aerodynamic efficiency of a BLI propulsor (full curve, with dots as CFD data points) as compared to the estimated theoretical efficiency (red curve).

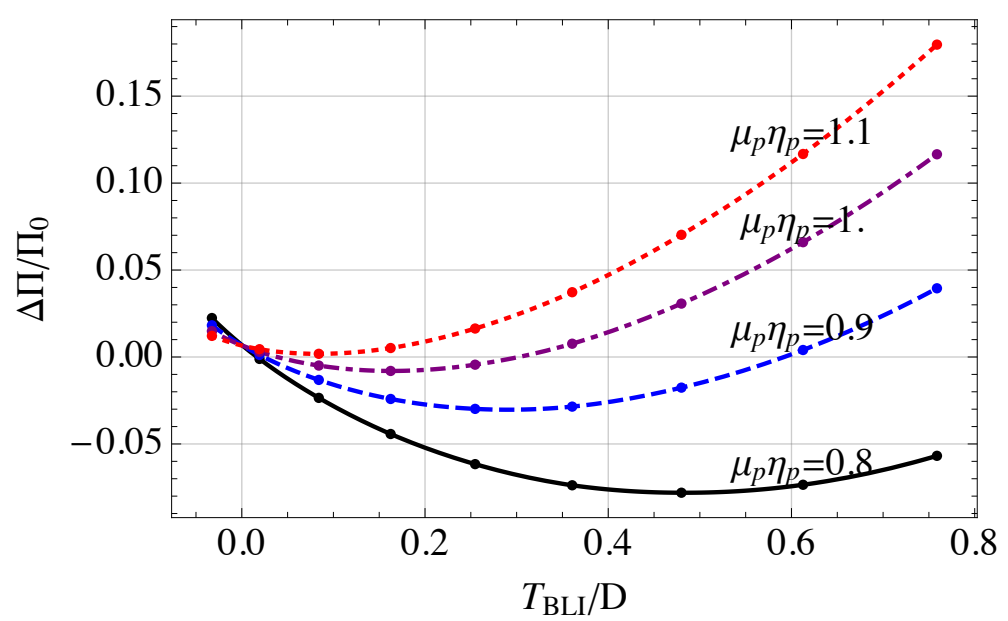

Figure 6. CFD computed aerodynamic power demand gain $\Delta \Pi$ of a BLI prop for different values of main propulsive efficiency (dots as CFD data points). 
without the BLR turbine was first evaluated for drag. After adding the turbine geometry, CFD cases for different angular velocities $\tilde{\omega}_{B L R} \in\{5,6,7, \ldots, 13\}$ were analyzed. The efficiency as given in equation (67) is plotted in figure 7. One can indeed see that the installed efficiency of the turbine is above unity due to its beneficial effect on body skin friction.

The effect of the turbine on overall configuration efficiency measured through overall power consumption is given in figure 8. The energy conversion efficiency was chosen as $\mu_{B L R}=1$. Here we see the opposite effect to the BLI prop; the more efficient the main mode of propulsion, the more beneficial is the effect of the turbine, as the additional braking force from the turbine can be efficiently offset through propulsion.

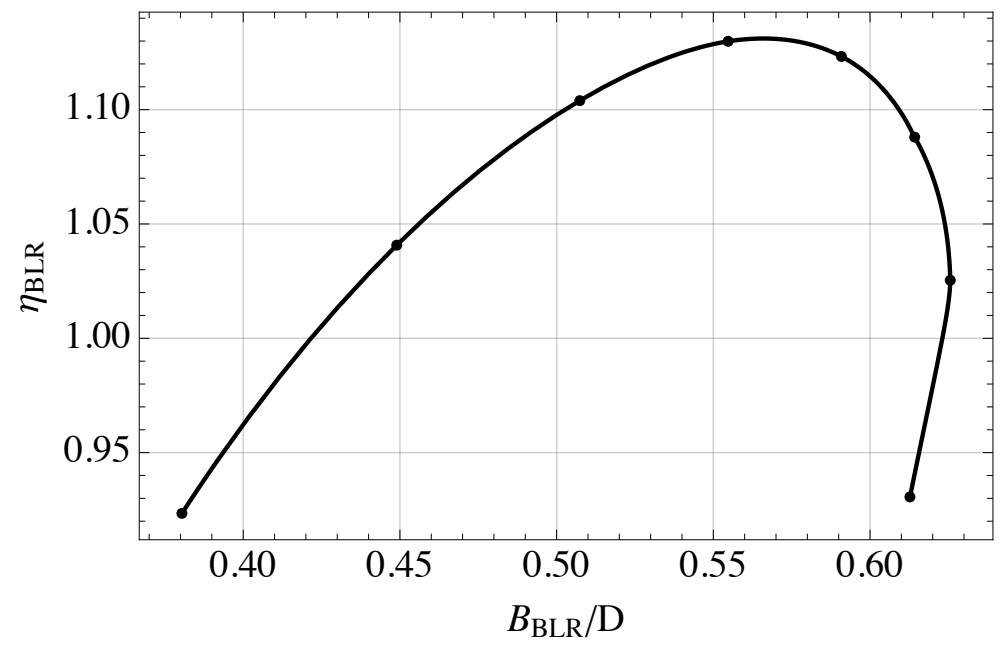

Figure 7. CFD computed aerodynamic efficiency of a BLR turbine, with dots as CFD data points.

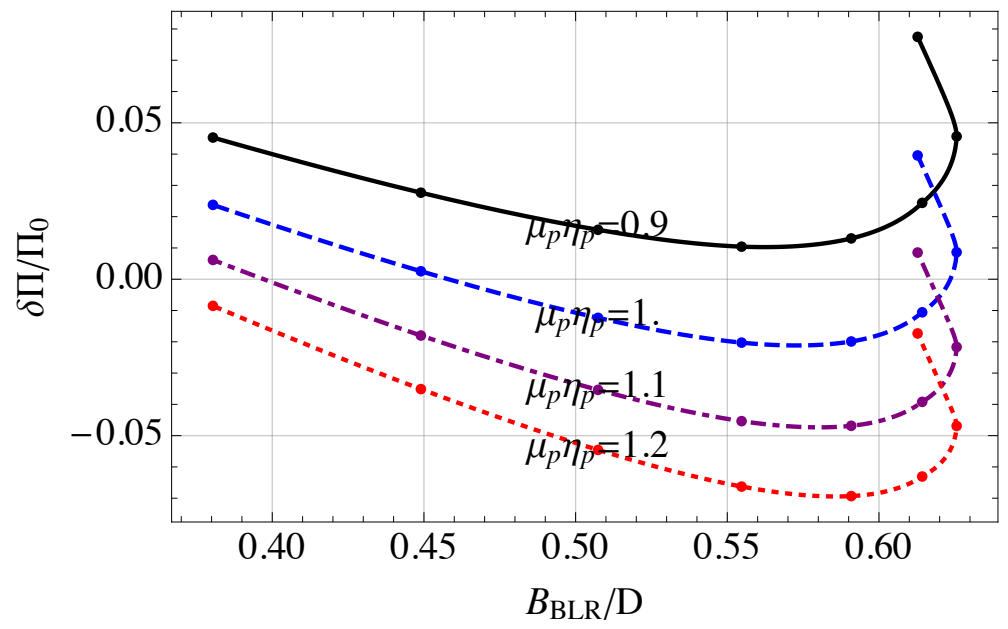

Figure 8. CFD computed aerodynamic power demand gain $\delta \Pi$ of a BLR turbine for different values of main propulsive efficiency (dots as CFD data points).

A configuration with simultaneous installation of both the BLR turbine and the BLI propeller is expected to show not only the contributions of individual components, but also an additional mutual benefit, as the propeller is operating in the boundary layer that has been previously slowed down by the turbine, which is expected to increase propeller efficiency.

The simultaneous case was computed for all combinations $\tilde{\omega}_{B L R}, \tilde{\omega}_{B L I} \in\{7.5,10,12.5\}$, and an interpolation of horizontal force and required powers was taken over all the cases. The resulting gains in power consumption as a function of non-dimensional angular velocities are given as a contour plot in figure 9. The assumed main propulsive efficiency was chosen $\mu_{p} \eta_{p}=1$. For the configuration studied, up to 5 percent gains are possible in aerodynamic power needed. The case of zero net thrust gain from turbine and propeller 
combination is particularly interesting as the gains do not depend on the main propulsive efficiency. Another interesting example is the zero total combined power, as in this case the propeller and turbine can be linked mechanically in ways that minimise power conversion losses.

A comparison can be made between the overall benefits of a combined configuration and the summed benefits of individual BLI propeller and BLR turbine configurations. The result of adding the thrust and power demand changes from individual cases and expressing them as a change in power demand is given in figure 10 for the same conditions as in figure 9. One may observe that the efficiency gain from separate contributions is less than when the propeller and turbine are working in unison, indicating that there exists a favourable interaction between the two components.

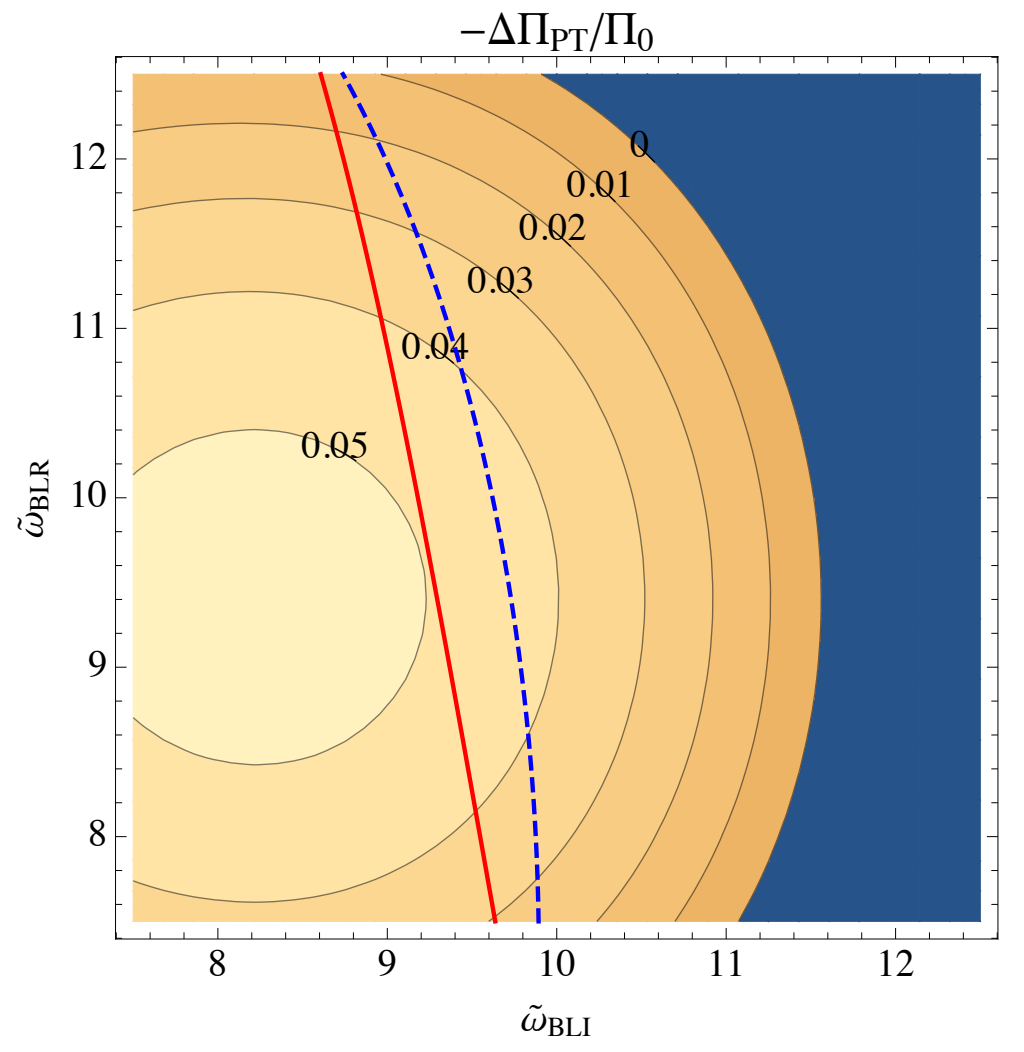

Figure 9. CFD computed aerodynamic power demand gain $-\Delta \Pi_{P T} / \Pi_{0}$ for a combination of a BLR turbine and a BLI propeller as a function of dimensionless angular velocities. The full red curve corresponds to the case of overall zero thrust change with respect to the base configuration, the blue dashed curve represents the case of zero total combined power on propeller and turbine.

\section{Conclusions}

The main point of this paper is that the benefits of aerodynamic efficiency of various propulsion or retardation devices can only be properly studied in the context of a complete configuration. Benefits of an added device can only be obtained if its efficiency exceeds the efficiency of the other installed devices. The condition of optimal power distribution amongst the devices for best energy efficiency is that all the propulsive devices should have the same differential energy efficiency, or its inverse value in the case of retardation devices.

We give a simple closed form model that captures the characteristics of boundary layer immersed propellers that can be used in simple preliminary analyses of such a device. We show that its benefits depend only on the total pressure loses in the captured boundary layer and not on the local flow characteristics of where the propeller is installed.

We introduce the concept of the boundary layer retardation turbine and confirm its benefits using CFD. We demonstrate that the BLI propeller analysis using CFD is in accordance with the proposed closed model, and, furthermore, that there exists a positive interaction between a BLR turbine and a BLI propeller when 


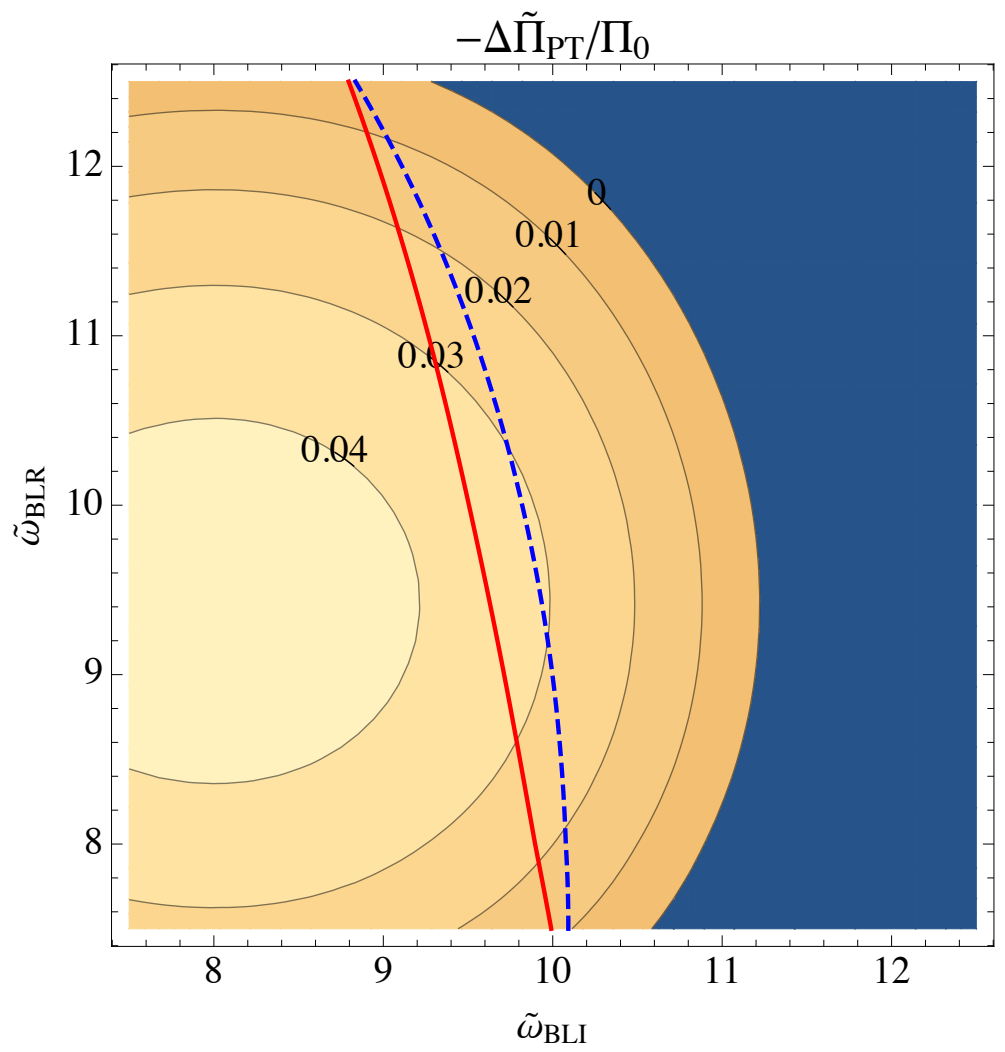

Figure 10. CFD computed aerodynamic energy gain $-\Delta \tilde{\Pi}_{P T} / \Pi_{0}$ added from separate BLR turbine and BLI propeller cases as a function of dimensionless angular velocities. The full red curve corresponds to the case of overall zero thrust change with respect to the base configuration, the blue dashed curve represents the case of zero total combined power on propeller and turbine. 
installed simultaneously. Even a non-optimised configuration shows power balance benefits. Further work is planned to optimise the geometries and to provide further theoretical insight into the BLR turbine.

\section{Acknowledgements}

Funding support was provided for this research by NASA Langley Research Center through the OnDemand Mobility exploratory studies, as part of the Transformational Aviation Concepts Program. Additional technology and concept studies looking at Thin-Haul market application of Distributed Electric (DEP) propulsion integration strategies have been performed by Joby Aviation and others under the guidance of Mark Moore, Ken Goodrich, Michael Patterson, and Bill Fredericks.

\section{References}

\footnotetext{
${ }^{1}$ Welstead J., and Felder J. L., "Conceptual Design of a Single-Aisle Turboelectric Commercial Transport with Fuselage Boundary Layer Ingestion", 54th AIAA Aerospace Sciences Meeting, AIAA SciTech, (AIAA 2016-1027).

${ }^{2}$ Seitz A., and Gologan C., "Parametric Design Studies for Propulsive Fuselage Aircraft Concepts", CEAS Aeronautical Journal, Vol. 6, Iss. 1, March 2015, pp. 69-82.

${ }^{3}$ Kaiser S., Grenon R., Bijewitz J., Prendinger A., Atinault O., Isikveren A.T., and Hornung M., "Quasi-Analytical Aerodynamic Methods For Propulsive Fuselage Concepts," 29th International Congress of the Aeronautical Sciences (ICAS), St. Petersburg, Russian Federation, September 7-12, 2013.

${ }^{4}$ Bijewitz J., Seitz A., Isikveren A. T., and Hornung M., "Multi-Disciplinary Design Investigation Of Propulsive Fuselage Aircraft Concepts", Aircraft Engineering and Aerospace Technology: An International Journal, Vol. 88, Iss. 2, 2016, pp. 257 267.

${ }^{5}$ Isikveren A.T., Seitz A., Bijewitz J., Hornung M., Mirzoyan A., Isyanov A., Godard J.-L., Stckl S., van Toor J., "Recent Advances In Airframe-Propulsion Concepts With Distributed Propulsion", 29th International Congress of the Aeronautical Sciences (ICAS), St. Petersburg, Russian Federation, September 7-12, 2013.

${ }^{6}$ Isikveren A. T., Mirzoyan A., Isyanov A., Grenon R., Atinault O., Godard J.-L., and Stckl S., "Distributed Propulsion And Ultra-High By-Pass Rotor Study At Aircraft Level," The Aeronautical Journal, Vol. 119, No. 1221, November 2015.

${ }^{7}$ Stoll A.M., Bevirt J., Moore M.D., Fredericks W.J., and Borer N.K., "Drag Reduction Through Distributed Electric Propulsion", 14th AIAA Aviation Technology, Integration, and Operations Conference, AIAA Aviation, (AIAA 2014-2851), 2014. $74-82$.

${ }^{8}$ Smith, L.H., "Wake Ingestion Propulsion Benefit," Journal of Propulsion and Power, Vol. 9, No. 1, Jan.-Feb. 1993, pp.

${ }^{9}$ Drela, M., "Power Balance in Aerodynamic Flows," AIAA Journal, Vol. 47, No. 7, July 2009, pp. 1761-1771.

${ }^{10}$ Goldschmied, F.R, "Aerodynamic Design Of Low-Speed Aircraft With A Nasa Fuselage/Wake-Propeller Configuration," Aircraft Systems, Design and Technology Meeting Dayton, OH, U.S.A. (AIAA 86-2693), 1986.

${ }^{11}$ Ormiston R.A., "On the Definitions of Rotor and Rotorcraft Power and Performance", AHS International 69th Annual Forum \& Technology Display, Phoenix, AZ, May 21-23, 2013.
} 